This item was submitted to Loughborough's Research Repository by the author.

Items in Figshare are protected by copyright, with all rights reserved, unless otherwise indicated.

\title{
Redundant and decentralised directory facilitator for resilient plug and produce cyber physical production systems
}

PLEASE CITE THE PUBLISHED VERSION

http://dx.doi.org/10.1007/978-3-319-51100-9_7

\section{PUBLISHER}

(C) Springer

VERSION

AM (Accepted Manuscript)

\section{PUBLISHER STATEMENT}

This work is made available according to the conditions of the Creative Commons Attribution-NonCommercialNoDerivatives 4.0 International (CC BY-NC-ND 4.0) licence. Full details of this licence are available at: https://creativecommons.org/licenses/by-nc-nd/4.0/

\section{LICENCE}

CC BY-NC-ND 4.0

\section{REPOSITORY RECORD}

Pascoa, Flavio, Ivo Pereira, Pedro Ferreira, and Niels Lohse. 2019. "Redundant and Decentralised Directory Facilitator for Resilient Plug and Produce Cyber Physical Production Systems”. figshare.

https://hdl.handle.net/2134/23332. 


\title{
Redundant and Decentralised Directory Facilitator for Resilient Plug and Produce Cyber Physical Production Systems
}

\author{
Flávio Páscoa ${ }^{1}$, Ivo Pereira ${ }^{2}$, Pedro Ferreira ${ }^{2}$, Niels Lohse ${ }^{2}$ \\ ${ }^{1}$ Everis, Consulting, IT \& Outsourcing Professional Services, Portugal \\ flavio.pascoa@everis.com \\ ${ }^{2}$ EPSRC Centre for Innovative Manufacturing in Intelligent Automation, Wolfson School of \\ Mechanical, Electrical and Manufacturing Engineering, Loughborough University, UK, \\ \{i.pereira, p.ferreira,n.lohse\}@lboro.ac.uk
}

\begin{abstract}
Cyber Physical Production Systems (CPSS) build on the concept of "Plug-and-Produce" aiming to deliver truly agile production systems. These systems are modular by nature and can be adapted based on changing requirements. One of the challenges in this domain is how to store and propagate information within CPPS. In this paper, a Redundant and Decentralised Directory Facilitator will be introduced to provide the capability to store and broadcast the existing system assembly capabilities. Additionally, this solution will provide redundancy and delocalization of the assembly capabilities information. The model used is described, as well as interactions, behaviours and deployment strategies. Finally a validation scenario is presented and conclusions are presented.
\end{abstract}

\section{Introduction}

The need for agile production systems has resulted in creation of Cyber Physical Production Systems (CPPS) concept. This concept builds on the "Plug-andProduce" concept which uses the modular concept for production systems making then easy to configure and reconfigure [1]. The equipment modules are cyber physical units that are able to independently operate and interact with other units. One of the most promising implementations for CPPS is the use of Agent technology as a means to embed the intended intelligence to each individual module and allow distributed control in a networked system [1]. This technology provides the needed dynamic control capabilities as well as the necessary decoupling and communication between modules. This means one can clearly represent each assembly capability inside the control agent architecture as shown in [3].

A method to keep track, organize and disseminate the existing assembly capabilities through the system is then essential to achieve optimal system functionality and performance. This method will provide the information about all the exist- 
ing system assembly capabilities and available modules to all the agents in the environment, which is critical for the emergent behaviour of the system.

One solution to accomplish this is a dedicated repository, where the information about all the available assembly capabilities and modules can be stored, grouped and organized, such as the JADE platform Directory Facilitator (DF) [4]. JADE is the most common agent platform and has been widely used in the modular assembly system domain [1]. The use of DF as part of the JADE platform is a centralized service. The service has some limitations regarding the exchanged message content, since it was designed to be quite generic, and therefore may not provide optimal solutions for CPPS. Furthermore, having this centralized service may cause issues in performance when system is scaled up as the DF has a limit of 100 as a response to a query [4]. More critically the use of a centralized approach can cause bottlenecks when a considerable amount of requests are being made. This will constitute a severe problem to the overall MAS performance, as delays on the repository response propagate to the assembly process execution.

Another important consideration is that a central service repository has a single point of failure, this means upon problems or malfunctions the CPPS will stop working. This means that the system would not be resilient to problems, as it would require some intervention with high potential of the system having to be stopped. In fact the use of a central approach would be contra intuitive to the plugand-produce concept which is the basis for CPPS.

This paper reports on a Redundant and Decentralised Directory Facilitator for Resilient as an extension of the available DF. This agent is called Capability Dissemination Agent (CDA) and will provide agents the necessary information to CPPS agents aiming at delivering resilient behaviour.

This paper is organised as follows: section 2 specifies the requirements for the CDA; section 3 describes the CDA model, with details about interactions, behaviours and deployment strategies; section 4 presents the validation study; and section 5 draws some conclusions.

\section{Capability Dissemination Agent Requirements}

An effective way of providing and organizing all the assembly capabilities available in a system is critical for enabling CPPS. These assembly capabilities are representations that are used within the control environment as triggers for the assembly processes. The use of the Skill concept [3] provides an established formalization for this representation. This is the first step for the creation of a working CPPS, therefore each CDA needs to contain this information and be able to provide it upon request. However, availability of information for the modules does not guarantee a resilient behaviour. Thus, it is proposed that multiple CDAs be present in any given system, making the capability dissemination a distributed process. Therefore, a method for the CDA to store and broadcast these Skills to other CDAs is then required. 
Due to the large amount of information that is expected to be stored, quick mechanisms to store the information and access it are needed. This will ensure performance targets can be achieved, since a slow CDA response to requests will cause delays on the system functionality.

The CDA must provide a method for other CDA's and other agents in the system to know when a CDA is introduced or removed. This ensures the "life beat" of all elements in the system, which is critical to deliver highly dynamic and agile systems. Additionally, this ability should to be independent of the platform where the CDA is launched, as one expects multiple platforms to exist in a CPS environment. This poses another implicit requirement which is the dissemination of when modules are added or removed across all platforms. Only with those discovery mechanisms can updated Skill information can be maintained and distributed across the system to deliver a resilient behaviour.

Due to the usage of agent technology, interactions between all the CDA and other agents must be defined. This would allow them to communicate with one another, allowing them to cooperate to achieve their individual objectives.

\section{Capability Dissemination Agent Model}

In a CPPS using agent technology, the assembly capabilities are executed by the hardware modules present in the system. These modules are independent having no knowledge about other modules and their capabilities. If each individual module capabilities are propagated through the system to all its existing members, an enormous number of messages would be exchanged, which could cause undesirable system underperformance.

A place to gather and organize the distributed assembly capabilities available in the system is then required. The proposed CDA is then used as an assembly capability gathering point, organizing and structuring the information, while propagating it through the system. A CDA will then have the information about the registered local capabilities. However, several CDAs can then be present. Each CDA must also know the local capabilities registered in all the other CDAs. This is obtained by regular information exchange between CDAs. Each CDA will then have the knowledge about all the assembly capabilities available throughout the system.

During the CDA life cycle, it needs to always maintain updated information about the entire system assembly capabilities. Only then can each CDA maintain an accurate view of the assembly capabilities present in the system.

To establish the CDA model, one needs to know what interactions are needed. The need to clearly enumerate each of the available relations and interactions arises so that its behaviours can be defined. Also, for this model, the Skill concept will be used to represent the assembly capabilities [5]. The interactions that may occur with the CDA can be seen in Fig. 1.

Any agent in the environment can register or deregister Skills within the CDA. Queries about existing Skills providers can also be made. The CDA will broadcast 
a message to the system informing it is born when he is first launched and a death message when he leaves the system. Periodic messages regarding the internal information status of the CDA will also be broadcasted.

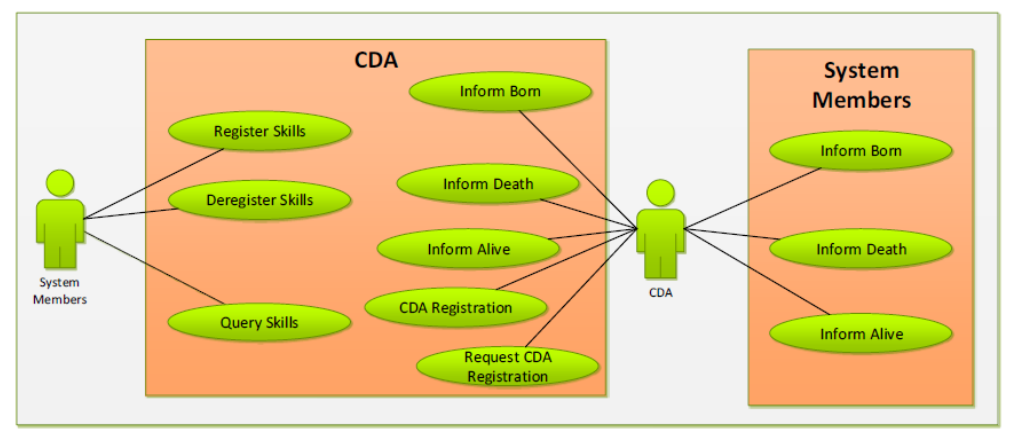

Fig. 1. CDA use cases overview

These define the possible interactions with the CDA. Its analysis will provide insight into all the required protocols. These protocols are the basis for creating an agent behaviour model which uses the protocols to achieve the agent objectives. Only with a clear and structured agent behaviour model will it be possible to create, implement and run the intended CDA. The first step to obtain the wanted model is to define the internal information each CDA needs to hold.

\section{Capability Dissemination Agent (CDA) Interactions and Behaviours}

All the interactions that may occur with the CDA need to be defined so that the proposed solution is stable and scalable. These interactions are mainly made using agent communication mechanisms which will be defined based on FIPA [6]. FIPA covers generic agent interactions which will significantly facilitate the development of the communication methods. For each interaction to be properly made, protocols need to be established. These protocols will provide the means for each member in the environment to interact with the CDA. FIPA establishes in its message contents the necessary information for a reply to be sent to the specific agent. The remaining message content is specific to each message and is defined accordingly.

- CDA Born Protocol - Consists in a broadcasted message to the network, specifying that the agent is alive;

- CDA Registration Protocol - Consists in a registration message to the existing CDAs, informing that it is in their neighbour;

- CDA Request Registration Protocol - Due to the dynamic environment, to request another CDA registration, another message is sent. After this message, the CDA Registration Protocol is used to finish this protocol; 
- CDA Status Protocol - During a CDA life cycle periodic messages will be exchanged between all the CDA in the system. This will allow them to know about each other internal information status as well as their existence;

- CDA Request Status Protocol - When for some reason a CDA needs to know other CDA internal information, it will use this protocol to obtain it;

- CDA Termination Protocol - When a CDA has a controlled exit of the system, it will broadcast a message informing it. A message with the same characteristics as the CDA Born message will be broadcast to the system;

- Skill Registration Protocol - When an agent knows how to execute some Skills and wants other agents know about it, it will register these Skills in the local CDA;

- Skill Deregistration Protocol - Same as above but with the objective to deregister skills in the local CDA;

- Skill Query Protocol - When a member needs to know which agent can perform a Skill, it will query the local CDA using this protocol.

Having the internal information structure defined, and also how each CDA will interact with other CDAs and other members in the system, it is now possible to create the behaviours models to obtain the wanted CDA objectives.

The CDA requires a mechanism to broadcast messages to the system. Its behaviours can then take advantage of this functionality. Each CDA is wanted to be functioning independently from each other. Each CDA will be associated with a different main container so that if one fails, the others can still keep functioning. A CDA registered in a JADE main container [4] cannot send messages to agents registered in others without previously knowing their individual address and names.

A mechanism so that a CDA can broadcast messages to agents in other containers is defined. This will be obtained using the Ethernet UDP Protocol [7]. It will provide a good performance approach as it does not requires connections to be established and maintained through the system. While the UDP protocol is not $100 \%$ reliable, its usage within a controlled environment will prove itself to be robust enough, as messages very rarely are lost. For this UDP broadcast to be functional, all the system members will be part of the same network as well as known the Group Port that the messages are sent and received through. All the agents that want to receive these messages must be listening to this defined port. Only with those prerequisites will allow that each message sent this way can be correctly received. This will allow the wanted message to reach every agent in the system, present in remote containers or in the local container, so long as agents are listening.

\section{Capability Dissemination Agent Deployment Strategy}

The CDA deployment is defined and adjusted considering the analysed system. In an extreme situation, a CDA for each module can be created. However, this implies a massive communication load. On the other side if only one CDA is present, then the system would not provide the necessary robustness. The deployment in 
the system needs to balance between the message load and the level of redundancy in the system. An overview of the CDA deployment strategies is shown in Fig. 2.

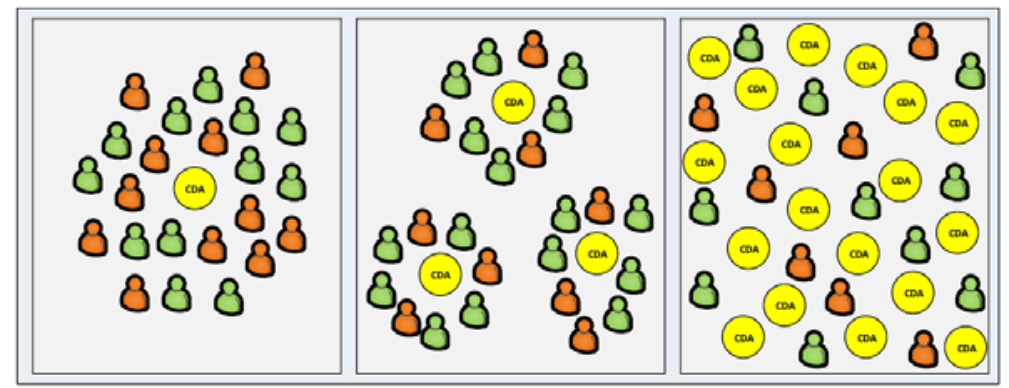

Fig. 2. CDA deployment strategies overview

In the figure three strategies are considered. The left strategy has one CDA for the entire system. This will represent the central repository approach that in the case of failure will bring the MAS to a complete stop. The middle strategy has several clusters formed having a CDA representing them. This will reduce the number of exchanged messages across the system but at the same time providing some system redundancy. The right strategy has a considerable amount of CDAs along with other agents. This will guarantee the module level redundancy but will again increase the number of messages exchanged between the existing CDA and make meaningless the use of CDA.

\section{Capability Dissemination Agent Adaptability Validation}

The initial validation for the CDA functionality was obtained in the previous experimental scenarios, as the CDA was used to store the system existing Skills, and diffusing then through the system. To validate the re-adaptation capabilities of the CDA, two stations will be used along with an external computer terminal.

Two CDAs will be initially launched, one in each of the two workstations. The equipment modules will be represented as Resource Agents (RA), which are also launched for each one of the stations. Afterwards, another CDA will be launched in the external computer without any RA associated with it. Its sole objective will be to display the information stored in its internal defined tables, and with that, observe the CDA functionality. To be able to visualise the necessary functionalities, an interface to display these tables was created, as seen in Fig. 3.

After the initial synchronization and information exchange, all the assembly capabilities registered in each individual station CDA are known by the external CDA. Also, their registered local skill providers are known.

All the system existing capabilities are known by the CDA. Next, one station will be abruptly shut down. This will provide confirmation on the CDA capability 
to readapt to partial system breakdowns. All the information regarding that specific CDA was no longer available once the CDAs synchronized. The information that is expected to disappear is shown highlighted by a red rectangle in Fig. 3.

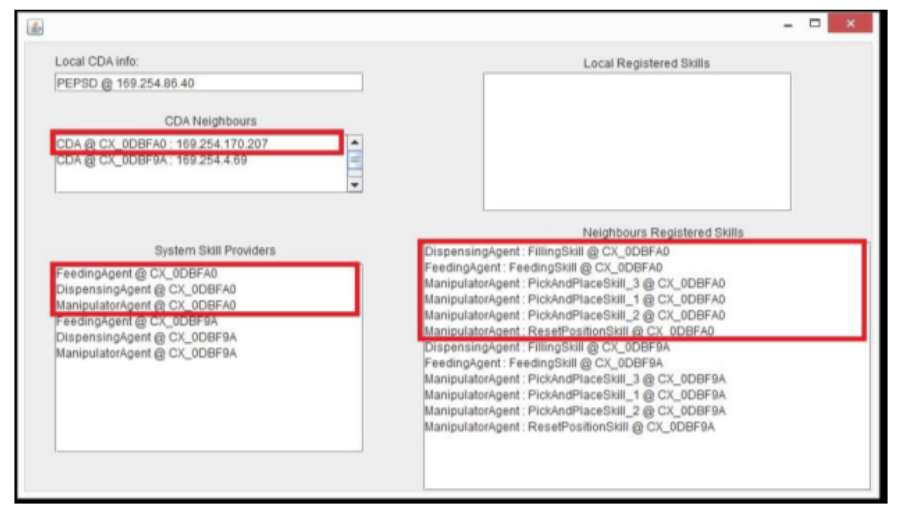

Fig. 3. CDA tables information display before neighbour CDA removal

The capabilities and skill providers associated with the removed station were removed from the CDA tables as expected, and can be confirmed in Fig. 4, since the top of the table as changed. The reintroduction highlighted in red in Fig. 4, is achieved after the station is reintroduced to the system.

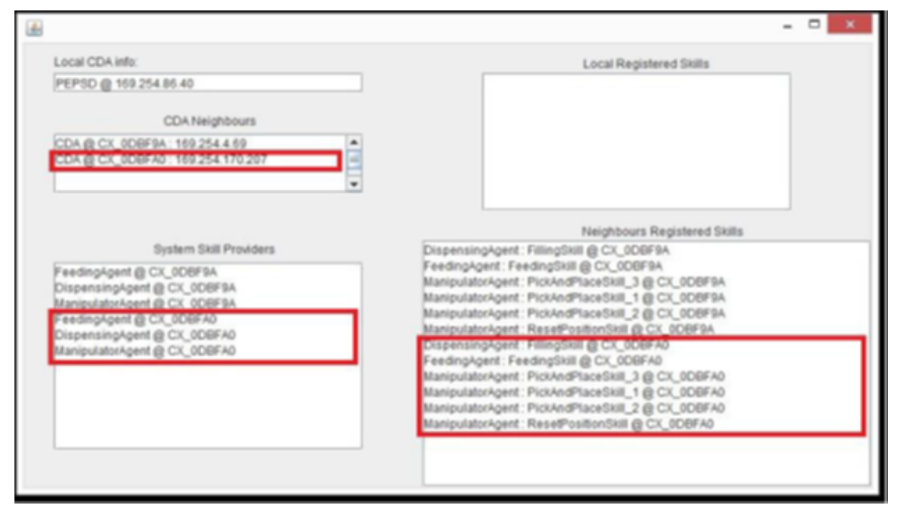

Fig. 4. CDA tables information display after neighbour CDA reconnection

As observed in the figure, highlighted by the red rectangle, the capabilities from the new introduced system were correctly propagated and its existence acknowledged by the external CDA.

This experimental scenario validates the proposed CDA model providing the assembly capabilities correct propagation and re-adaptation to changes. Also, de discovery mechanism fulfilled its task, as the neighbours know when a new CDA was introduced. 


\section{Conclusion}

The use of a centralized approach for the storage of assembly capabilities in the context of CPPS is seen as a drawback for the realization of this concept. However it is clear the information needs to be stored and readily available for this concept to work. This paper proposes multiple delocalized CDA to maintain the information of all the system capabilities while being capable to readapt to changes. Each CDA can communicate across different platforms and keep track of other CDAs, along with their registered Skills. Redundancy and persistence emerges from the interaction of this collaborative behaviour. It increases the system robustness by not having the central capability repository, which allows the system to keep functioning even if part of it goes down. This approach proves very resilient to changes in the system and also addresses the challenges of scalability as it does not have any limits for the number of modules in the system. Moreover, because these agents are delocalised these could be distributed to cater for computational resource limitations, without impact to the operation of the system.

The deployment strategies for this approach is discussed, however more work needs to be done in order to establish what is the ideal distribution of CDAs in a system taking into account the system scale and topology, as well as performance requirements. Nevertheless, this paper demonstrates the first steps to achieve a redundant and decentralised directory facilitator for CPPS which is able maintain, broadcast and secure all available assembly capabilities in the system.

\section{Acknowledgments}

The authors acknowledge funding from the European Union's Horizon 2020 research and innovation programme under grant agreement No 680735 and support from the EPSRC Centre for Innovative Manufacturing in Intelligent Automation, in undertaking this research work under grant reference number EP/IO33467/1.

\section{References}

1. T. Arai, Y. Aiyama, Y. Maeda, M. Sugi and J. Ota, Agile Assembly System by "Plug and Produce”. CIRP Annals - Manufacturing Technology, 2000. 49(1): p. 1 - 4.

2. P. Ferreira, An agent-based self-configuration methodology for modular assembly systems, PhD thesis, University of Nottingham, 2011.

3. P. Ferreira, N. Lohse, M. Razgon, P. Larizza, and G. Triggiani, Skill Based Configuration Methodology for Evolvable Mechatronic Systems, in IECON 2012-38th Annual Conference on IEEE Industrial Electronics Society, pp. 4366-4371, 2012.

4. F. Bellifemine, G. Caire, T. Trucco, G. Rimassa, and R. Mungenast, Jade Administrator's Guide, JADE 4.0 ed. Boston, MA, 2010.

5. P. Ferreira and N. Lohse, Configuration Model for Evolvable Assembly Systems, 4th CIRP Conference on Assembly Technologies and Systems to be held on May 21-22, 2012, in Ann Arbor, Michigan, USA.

6. Fipa, FIPA ACL Message Structure Specification, Foundation for Intelligent Physical Agents, 2002.

7. A. S. Tanenbaum and D. Wetherall. Computer networks. Prentice Hall, 2011. 